\title{
VIEW-OBSTRUCTION PROBLEMS. II
}

\author{
T. W. CUSICK
}

\begin{abstract}
Let $S^{n}$ denote the region $0<x_{i}<\infty(i=1,2, \ldots, n)$ of $n$-dimensional Euclidean space $E^{n}$. Suppose $C$ is a closed convex body in $E^{n}$ which contains the origin as an interior point. Define $\alpha C$ for each real number $\alpha>0$ to be the magnification of $C$ by the factor $\alpha$ and define $C+\left(m_{1}, \ldots, m_{n}\right)$ for each point $\left(m_{1}, \ldots, m_{n}\right)$ in $E^{n}$ to be the translation of $C$ by the vector $\left(m_{1}, \ldots, m_{n}\right)$. Define the point set $\Delta(C, \alpha)$ by $\Delta(C, \alpha)=\left\{\alpha C+\left(m_{1}+\frac{1}{2}, \ldots, m_{n}+\frac{1}{2}\right)\right.$ : $m_{1}, \ldots, m_{n}$ nonnegative integers $\}$. The view-obstruction problem for $C$ is the problem of finding the constant $K(C)$ defined to be the lower bound of those $\alpha$ such that any half-line $L$ given by $x_{i}=a_{i} t(i=1,2, \ldots, n)$, where the $a_{i}(1<i<$ $n)$ are positive real numbers, and the parameter $t$ runs through $[0, \infty)$, intersects $\Delta(C, \alpha)$.

The paper considers the case where $C$ is the $n$-dimensional cube with side 1 , and in this case the constant $K(C)$ is known for $n<3$. The paper gives a new proof for the case $n=3$. Unlike earlier proofs, this one could be extended to study the cases with $n>4$.
\end{abstract}

1. Introduction. The view-obstruction problems defined in the abstract were first introduced in [2]. In this paper we only consider the case where the closed convex body $C$ in $E^{n}$ is the $n$-dimensional cube with side 1 . We use the notation $\lambda(n)$ for the constant $K(C)$ in this case.

For any real number $x$, let $\|x\|$ denote the distance from $x$ to the nearest integer. The evaluation of $\lambda(n)$ can be thought of as a problem in Diophantine approximation, since we have

$$
\frac{1}{2} \lambda(n)=\sup \min _{0<x<1} \max _{1<i<n}\left\|w_{i} x-\frac{1}{2}\right\|,
$$

where the supremum is taken over all $n$-tuples $w_{1}, \ldots, w_{n}$ of positive integers. If we define

$$
\kappa(n)=\inf \max _{0<x<1} \min _{1<i<n}\left\|w_{i} x\right\|,
$$

where the infimum is taken over all $n$-tuples $w_{1}, \ldots, w_{n}$ of positive integers, then since $\left\|w_{i} x\right\|=\frac{1}{2}-\left\|w_{i} x-\frac{1}{2}\right\|$, we have $\lambda(n)=1-2 \kappa(n)$ for each $n>2$. It will be convenient in the rest of the paper to concentrate on the problem of evaluating $\kappa(n)$.

The problem of evaluating $\lambda(n)$ is equivalent to the following: Suppose the unit cube in $E^{n}$ has faces which reflect a certain particle, and consider any motion of the particle, starting in a corner of the cube and not entirely contained in a hyperplane of dimension $n-1$. What is the side length of the largest subcube,

Received by the editors January $25,1980$.

1980 Mathematics Subject Classification. Primary 10F10; Secondary 10E99.

() 1982 American Mathematical Society 0002-9939/82/0000-0006/\$02.00 
centered in the unit cube, with the property that there exists such a motion of the particle which does not intersect the subcube? Plainly the largest such side length is $\lambda(n)$.

The corresponding problem, if the condition that the particle start in a corner is omitted, can be treated by methods entirely different from those in this paper. This has been done by I. J. Schoenberg [4], who solved this problem in every dimension; he showed that the largest subcube in dimension $n$ has side $1-n^{-1}$.

The natural conjecture for the value of $\lambda(n)$ is $(n-1) /(n+1)$ (as stated in [2, p. 166]). This is because Dirichlet's box principle gives $\max _{0<x<1} \min _{1<i<n}\|i x\|=$ $1 /(n+1)$, so $\kappa(n)<1 /(n+1)$, and it is reasonable to conjecture that equality holds. It is this conjecture that is proved in this paper for $n<3$.

The case $n=2$ is very simple. The case $n=3$ is considerably more complicated, but several proofs have previously been published (Betke and Wills [1], Cusick [2, 3]), and another one is given here. The proof in this paper, unlike the earlier proofs, has the advantage that it can be extended to study the cases with $n>4$; however, the argument is then no longer elementary. The author hopes to return to this question elsewhere.

2. Another proof that $\kappa(3)=\frac{1}{4}$. By $(1)$, in order to show that $\kappa(n)=1 /(n+1)$ it is enough to prove that given any $n$-tuple $w_{1}, \ldots, w_{n}$ of positive integers with the property that, for any integers $m$ and $q$,

$$
\left\|w_{i} q / m\right\| \leqslant 1 /(n+1) \text { for some } i, 1 \leqslant i \leqslant n,
$$

there exists some pair $m, q$ such that (2) does not hold if $\leqslant$ is replaced by $<$.

If we assume (as we may with no loss of generality) that $w_{1}, \ldots, w_{n}$ have no common prime factor, then we would expect that there are only finitely many $n$-tuples $w_{1}, \ldots, w_{n}$ such that (2) holds for any $m$ and $q$. Further, we might hope that by considering only finitely many values of $m$, we could identify all of these $n$-tuples, and so reduce the determination of $\kappa(n)$ to a finite calculation. It is easy to carry out this procedure when $n=2$, and so prove $\kappa(2)=\frac{1}{3}$. In the remaining portion of this paper, we show that the procedure can also be successfully carried out when $n=3$.

For the rest of this section, we take $n=3$ and suppose $w_{1}, w_{2}, w_{3}$ is a triple of integers, having no common prime factor, such that (2) holds for any integers $m$ and $q$. Our goal is to show that we can always find a pair of integers $m$ and $q$ such that

$$
\min _{1<i<3}\left\|w_{i m} \frac{q}{m}\right\|>\frac{1}{4} \text {. }
$$

If $w$ is odd, then $\left\|\frac{w}{2}\right\|=\frac{1}{2}$, so we can assume that at least one of the $w_{1}$ is even. It is easy to prove that (3) holds if exactly two of the $w_{i}$ are even. First suppose that $w_{1}=2^{k} a, w_{2}=2^{k} b$ and $w_{3}=c$, where $a, b, c$ are odd and $k$ is $>1$. If we take $m=2^{k+1}$ and choose $q$ to be any odd integer such that $q c \equiv 2^{k}+1 \bmod 2^{k+1}$, then (3) holds. Next suppose that $w_{1}=2^{j+k} a, w_{2}=2^{k} b$ and $w_{3}=c$, where $a, b, c$ are odd and $j, k$ are $\geqslant 1$. We take $m=2^{j+k+1}$ and will take $q$ to be odd, so 
$\left\|w_{1} q / m\right\|=\frac{1}{2}$. In order to specify $q$, we first choose an odd $q_{0}$ such that $b q_{0} \equiv t$ $\bmod 2^{j+1}$, where $t$ is an odd integer satisfying $\left\|t / 2^{j+1}\right\|>\frac{1}{4}$. We define $q$ to be $q_{0}+2^{j+1} r$, where $r$ is chosen so that $\left\|w_{3} \frac{q}{m}\right\|>\frac{1}{4}$ (such a choice of $r$ is possible since changing $r$ by 1 changes $\left\|w_{3} \frac{q}{m}\right\|$ by $\left.\left\|c / 2^{k}\right\|\right)$. With this choice of $q$, (3) holds.

Now we suppose that exactly one of the $w_{i}$ is even, say $w_{1}=2^{k} a, w_{2}=b, w_{3}=c$, where $a, b, c$ are odd and $k$ is $\geqslant 1$. For this case we need the following elementary lemma.

LEMMA 1. For $u$ any odd integer and $n$ any integer $>3$, define $S_{n}(u)=S(u)=$ $\left\{\right.$ least positive residues $\bmod 2^{n}$ of odd $t$ satisfying $\left.\left\|t u / 2^{n}\right\|>\frac{1}{4}\right\}$. Then for any pair $u$, $v$ such that $1 \leqslant u, v<2^{n-1}$, we have

$$
S(u) \cup S(v)=\left\{\text { all odd } t \bmod 2^{n}\right\}
$$

if and only if $u+v \equiv 0 \bmod 2^{n-1}$.

Proof. The "if" part of the lemma is clear, since $u+v \equiv 0 \bmod 2^{n-1}$ and $1<u, v<2^{n-1}$ means $u+v=2^{n-1}$, so $\left\|t u / 2^{n}\right\|=\left\|\left(2^{n-1}-t\right) v / 2^{n}\right\|$. Since $t$ belongs to $S(u)$ if and only if $2^{n-1}-t$ does not belong to $S(u)$, we have (4).

To prove the "only if" part of the lemma, it is enough to show that $S(u)=S(v)$ cannot happen if $u \neq v$ and $1 \leqslant u, v<2^{n-1}$; for if (4) holds with $1 \leqslant u, v<2^{n-1}$, then $S\left(2^{n-1}-v\right)=S(u)$. Define

$$
M=\left\{\text { odd } m \text { satisfying } 2^{n-2}<m<3 \cdot 2^{n-2}\right\},
$$

so $r$ is in $S(u)$ if and only if $r u \equiv m$ mod $2^{n}$ for some $m$ in $M$. Thus $S(u)=S(v)$ means that the set $M$ is unchanged when the elements of $M$ are multiplied by $u^{-1} v$ and reduced $\bmod 2^{n}$; we use the notation $u^{-1} v M=M$ for this. We prove that if $x$ is any integer such that $x M=M$, then $x \equiv \pm 1 \bmod 2^{n}$. This will complete the proof of the lemma; for then $S(u)=S(v)$ implies either $u^{-1} v \equiv-1 \bmod 2^{n}$ (so $u+v \equiv 0$ $\bmod 2^{n}$, which is impossible if $1 \leqslant u, v<2^{n-1}$ ) or $u^{-1} v \equiv 1 \bmod 2^{n}$ (so $u=v$ ).

So we suppose $x M=M$ with $1<x<2^{n}$. Clearly this implies $x^{i} M=M$ for each $i=1,2, \ldots$ Let $d$ be the order of $x \bmod 2^{n}$; note $d$ is even since $d>1$ and $d$ divides $\phi\left(2^{n}\right)=2^{n-1}$. We have $\left(x^{d / 2}\right)^{2} \equiv 1 \bmod 2^{n}$, and the roots of $y^{2} \equiv 1 \bmod$ $2^{n}$ for $n \geqslant 3$ are $y \equiv 1,-1,2^{n-1}+1$ or $2^{n-1}-1$. We cannot have $x^{d / 2} \equiv 1 \bmod 2^{n}$ (this contradicts the definition of $d$ ) or $x^{d / 2} \equiv 2^{n-1} \pm 1 \bmod 2^{n}$ (for then $x^{d / 2} M=$ $M$, but the element $x^{d / 2}\left(2^{n-1} \pm 1\right) \equiv 1$ is not in $M$-contradiction). Hence $x^{d / 2} \equiv$ $-1 \bmod 2^{n}$; if $d$ is divisible by 4 this is impossible because $y^{2} \equiv-1 \bmod 2^{n}$ has no solutions. Hence $d=2$, so $x \equiv-1 \bmod 2^{n}$ and the proof is complete.

Now we turn to the proof of (3) for $w_{1}=2^{k} a, w_{2}=b, w_{3}=c$. We will choose $q$ odd and $m$ equal to either $2^{k+1}$ or $2^{k+2}$, so we may assume without loss of generality that $1 \leqslant b, c<2^{k+1}$. First suppose that $m=2^{k+2}$. If we can find an integer $q$ such that $q$ belongs to both $S_{k+2}(b)$ and $S_{k+2}(c)$ (using the notation of Lemma 1), then (3) holds. If no such $q$ exists, then by Lemma 1 with $n=k+2$ we have $b+c \equiv 0 \bmod 2^{k+1}$. This means $S_{k+1}(b)=S_{k+1}(c)$, so if we choose $q$ to be any integer in $S_{k+1}(b)$ and take $m=2^{k+1}$, then (3) holds. This finishes the proof that $\kappa(3)=\frac{1}{4}$. 


\section{REFERENCES}

1. U. Betke and J. M. Wills, Untere Schranken für zwei diophantische Approximations-Funktionen, Monatsh. Math. 76 (1972), 214-217.

2. T. W. Cusick, View-obstruction problems, Aequationes Math. 9 (1973), 165-170.

3. __ View-obstruction problems in n-dimensional geometry, J. Combin. Theory Ser. A 16 (1974), $1-11$.

4. I. J. Schoenberg, Extremum problems for the motions of a billiard ball. II. The $L_{\infty}$ norm, Nederl. Akad. Wetensch. Proc. Ser. A 79 = Indag. Math. 38 (1976), 263-279.

Department of Mathematics, State University of New York at Buffalo, Buffalo, New York 14214 